\title{
Simulation of influence of disturbance on the release of endogenous phosphorus in sediment of HAOHE River of Nantong, China
}

\author{
Xiu LU ${ }^{1}$, Jia $\mathrm{LI}^{1, \mathrm{a}}$ and Ping DUAN ${ }^{1}$ \\ ${ }^{1}$ School of Tourism and Geographical Sciences of Yunnan Normal University Yunnan, China
}

\begin{abstract}
There are less researches in the influence of disturbance of endogenous phosphorus release in sediment. The correlational analysis only takes the disturbance as one of the many factors in influencing the phosphorus release. It hasn't been able to make a deeper discussion from disturbance itself. In this paper, HAOHE River of Nantong, China is selected as study area. The whole process of disturbance is simulated in the laboratory. Disturbance intensity, time and depth is respectively simulated and analysed their effects on endogenous phosphorus release in sediment. The result shows that the external disturbance can significantly increase the release of endogenous phosphorus in sediment. From the time series of the experimental results, the release of phosphorus is not increased with the increasing time. The depth experiment has showed that the maximum phosphorus release is closest to the sediment and the closest to the water surface, while the phosphorus release in the position of middle depth is less.
\end{abstract}

\section{Introduction}

In the aspect of controlling the eutrophication of water body, the endogenous release of the sediment has been controversial. For the first time, foreign scholars put forward the endogenous release of water body in 1941. For a long time, the scientific research shows that N, P and other elements are the main factors that limit the growth of plankton, in which phosphorus the biggest factor leading to eutrophication among them. Nutrient elements, such as nitrogen and phosphorus, have important functions on primary productivity and nutrient level of water body. In recent years, the effects of sediment on the internal nutrient sources have been paid more and more attention in the control of exogenous phosphorus. At present, the release of endogenous phosphorus has been up to more than half a century. Studies show that the main factors affecting the release of endogenous phosphorus are environmental factors ${ }^{[1]}$. At home and abroad, scholars have carried out thorough study, most of the method is the release method. Various factors affect endogenous release, such as temperature, redox potential, $\mathrm{PH}$, disturbance, microbial action, the ratio of iron and phosphorus in sediment and the condition of aquatic plants ${ }^{[2]}$. Li Dapeng ${ }^{[3]}$ and others believe that the release of endogenous phosphorus has two kinds of static and dynamic release in some shallow water bodies. Relatively speaking, the release amount of the dynamic release is much more than the static one. So the dynamic release of endogenous phosphorus is more concerned. The factors which influence the large factors are the wind wave disturbance, the biological disturbance, the tidal current disturbance, the artificial disturbance and so on.

\section{Materials and methods}

\subsection{General situation of study area}

Nantong is known as "China's first modern city", with a long history and strong cultural heritage. It has a moat HAOHE river which has a thousand years of history, the spatial pattern of integrity and a very high historical value. But the HAOHE River also encountered many typical problems in the history. With the economic development, urban scale expansion and the increase intensity of the transformation of the old city in Nantong resulting in the residential and high-rise buildings continued warming. It is not only suffer from man-made destruction, industrial pollution caused by water black odor, the most serious time being the HAOHE River every day to bear nearly million tons of domestic sewage and industrial wastewater. Then the public likened HAOHE River being vividly "wash rice and vegetables in the 1950 s, deterioration of water quality in the 1960s, fish and shrimp are dead in the 1970s, eighties wash dirty toilet cover cannot be washed neatly in the 1980s ."Later in order to change this situation, local government and the people conduct a series of reform measures. Until today, the HAOHE River has successfully promoted to the state $5 \mathrm{~A}$ class tourist scenic spot. The HAOHE River line length of approximately $14.96 \mathrm{~km}, 1040$ acres of water surface, the widest at 215 meters, the mighty waves, like the lake, the narrowest point is only 10 meters which like

a Jia LI: keguigiser@163.com 
the drift ribbon. HAOHE River can be divided into the southeast, southwest and North River three sections. Nantong becomes a "water surrounds City, city surrounds water" city, which has a unique landscape. It was praised as "Oriental Venice" by some people. The HAOHE River water quality is gradually good, when the external pollution is under control, how the external disturbance will affect the HAOHE internal sediment pollution release? The author from August 2013 to April 2014 in the HAOHE River respectively in the vicinity of HAOHE River People's Park Bridge and North Bridge North HAOHE for sampling and testing.(As is shown in Figure1)

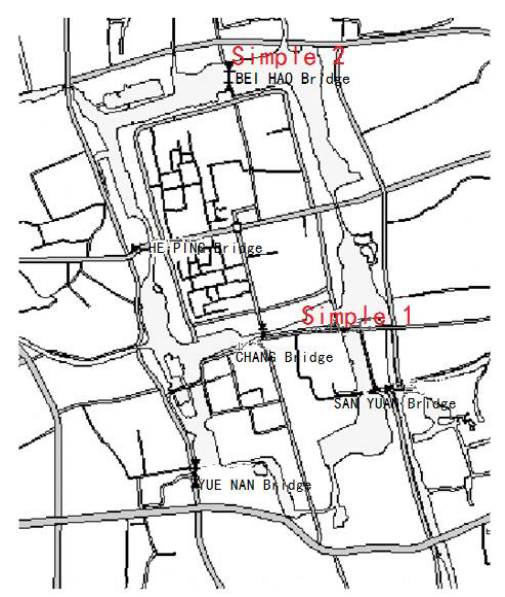

Figure1. Nantong HAOHE River ${ }^{[4]}$

Table1. List of sample points

\begin{tabular}{|c|c|c|}
\hline Number & $\begin{array}{l}\text { Geographical } \\
\text { coordinates }\end{array}$ & Location \\
\hline Sample point 1 & $32^{\circ} 00^{\prime} 56.19^{\prime \prime} \mathrm{N}$ & $\begin{array}{lr}\text { HAOHE } & \text { River } \\
\text { People's } & \text { Park } \\
\text { Bridge } & \\
\end{array}$ \\
\hline \multirow[t]{2}{*}{ Sample point 2} & $\begin{array}{l}120^{\circ} 51^{\prime} 57.529^{\prime \prime} \mathrm{E} \\
32^{\circ} 01^{\prime} 31.949^{\prime \prime} \mathrm{N}\end{array}$ & North \\
\hline & $120^{\circ} 51^{\prime} 36.999^{\prime \prime} \mathrm{E}$ & North HAOHE \\
\hline
\end{tabular}

\subsection{Reagent and instruments}

Main materials and reagents: Nantong HAOHE River mud sediment and overlying water; sulfuric acid: 1:1; potassium peroxydisulfate: $50 \mathrm{~g} / \mathrm{L}$ solution; ascorbic acid: $100 \mathrm{~g} / \mathrm{L}$ solution (store in a brown bottle reagent); molybdate solution: dissolve $13 \mathrm{~g}$ ammonium molybdate solution in $100 \mathrm{ml}$ of water. Dissolve $0.35 \mathrm{~g}$ antimony potassium tartrate in $100 \mathrm{ml}$ water. Metastasis after reagent slowly under constant agitation to ammonium molybdate solution adding $300 \mathrm{ml}$ sulfate $(1: 1)$, and tartaric acid potassium antimony solution and mixed evenly (store in brown bottles); phosphorus standard reserving solution: weigh $0.2197 \pm 0.001 \mathrm{~g}$ to 110 degrees centigrade and dried in a desiccator for two hours and put cold monopotassium phosphate. And then dissolve in water to $1000 \mathrm{ml}$ volumetric flask, add about $800 \mathrm{ml}$ water, add $5 \mathrm{ml}$ of sulfuric acid (1:1), dilute with water to marked line and mixed evenly, the solution in the glass bottle can be stored for at least six months; phosphorus standard using liquid: $10.0 \mathrm{ml}$ of phosphorus standard solution is transferred to a $250 \mathrm{ml}$ volumetric flask and water dilution to marked line and mixed evenly (prepare the solution when use that day). Main equipment: high pressure steam disinfection device; centrifuge; spectrophotometer; constant speed blender.

\subsection{Method}

\subsubsection{Analytical method}

Potassium peroxydisulfate dissolution: the sample $(25 \mathrm{ml}$ stoppered graduated tube with sample) was added $4 \mathrm{ml}$ of potassium peroxydisulfate, the stoppered graduated tube cover after plugged with a small piece of cloth and tight wire glass stopper, put placed in a large beaker was heated high-pressure steam sterilizer, until a pressure of $1.1 \mathrm{~kg} / \mathrm{cm}^{2}$, corresponding to a temperature of 120 degrees centigrade. And maintain heating was stopped after 30min. After the pressure gauge is approaching zero, remove and let cool. Then dilute with water to the marked line. Chromogenic: respectively, each part was added $1 \mathrm{ml}$ ascorbic acid solution into the digestion solution and mix, 30s later adding $2 \mathrm{ml}$ molybdate solution and mix well. Spectrophotometry measure: $15 \mathrm{~min}$ after standing at room temperature, optical path $30 \mathrm{~mm}$ cuvette, at a wavelength of $700 \mathrm{~nm}$, with water as a reference, to measure the absorbance. After deducting the absorbance of the blank test, inquire the phosphorus content from the working curve.

\subsubsection{Experimental method}

\subsubsection{Sample preprocessing}

In Nantong HAOHE River sample points with Gravity Sampler collected sediment into the laboratory in the bucket is about $500 \mathrm{~g}$. And in the same place it above the acquisition of overlying water 50L. Bring back to the lab, the first disturbance strength and phosphorus of the relationship between a set of experimental samples to 40 degrees centigrade oven drying and grinding the standby. The sediment barrel needn't dry and grind. PH between 7-8 and, the temperature maintain at 10-14 degrees, the relative humidity between the stability of the 60-80. It has little change, and it also play a very good condition for considering the only one factor which is disturbance.

\subsubsection{Sample handling}

(1) Disturbance strength group: Take the drying and grinding the $40 \mathrm{~g}$ samples into the volume of $1000 \mathrm{ml}$ cylindrical container, plus the cover water $800 \mathrm{ml}$. A total of two groups of experiments: A group only on the overlying water, B group of sediment and the overlying water with a constant speed mixer for continuous disturbance $12 \mathrm{~h}^{[5]}$. Change the disturbance intensity, that is, the performance of a certain gradient of speed, 
respectively is $100 \mathrm{r} / \mathrm{min}, 150 \mathrm{r} / \mathrm{min}, 200 \mathrm{r} / \mathrm{min}, 250 \mathrm{r} / \mathrm{min}$, $300 \mathrm{r} / \mathrm{min}, 350 \mathrm{r} / \mathrm{min}, 400 \mathrm{r} / \mathrm{min}$, and the interval is $50 \mathrm{r} / \mathrm{min}$. Each set of three groups of parallel samples, the error will be reduced to less than 0.05. (2) Time series group: The weighed returned to samples of about $2 \mathrm{~cm}$ in thickness, added to the overlying water to the bottom diameter is $16 \mathrm{~cm}, 27 \mathrm{~cm}$ high cylinder shaped container, the depth of the water remained in the $25 \mathrm{~cm}$. A total of three groups of experiments: A group only on the water, the B group on the overlying water and sediment does not disturb the $\mathrm{C}$ group of sediment and overlying water and disturbance, the disturbance group disturbance intensity is set to $200 \mathrm{r} / \mathrm{min}$ (because of its intensity of the maximum release amount). The time series are $1 \mathrm{H}, 3 \mathrm{~h}, 6 \mathrm{~h}$, 12h, 24h (one day), 72h (three days), by simulating a natural wind. Each set of three groups of parallel samples, the error will be reduced to less than 0.05. (3) Depth sequence group: The weighed returned to samples of about $2 \mathrm{~cm}$ in thickness, adding overlying water to the bottom diameter is $16 \mathrm{~cm}$, high $27 \mathrm{~cm}$ in cylindrical vessels. A total of three groups of experiments: A group only on the water, the B group on the water covered with sediment does not disturb the $\mathrm{C}$ group of sediment and overlying water and disturbance, the disturbance intensity of $200 \mathrm{r} / \mathrm{min}$, rotation time of $24 \mathrm{~h}$ (due to its release of the maximum time). The depth of the water samples are: $10 \mathrm{~cm}, 15 \mathrm{~cm}, 20 \mathrm{~cm}, 25 \mathrm{~cm}$. Each set of three groups of parallel samples, the error will be reduced to less than 0.05 .

\section{Result and analysis}

\subsection{Effects of disturbance intensity on phosphorus release from sediment}

As is shown in Figure 2, a horizontal coordinate indicates the speed, and the vertical coordinate indicates that the phosphorus release concentration (released amount is equal to the later phosphorus content minus the original phosphorus content). (The standard curve is: $\mathrm{R}^{2}=0.9999$ $\mathrm{y}=0.0204 \mathrm{x}+0.0011$ ) from the figure can be clearly known, at the speed of $200 \mathrm{r} / \mathrm{min}$, the maximum phosphorus release. After the release of phosphorus did not always increase, than $100 \mathrm{r} / \mathrm{min}$, emissions are not $400 \mathrm{r} / \mathrm{min}$, to $200 \mathrm{r} / \mathrm{min}$ increases to a maximum and also decreases, and in the min and the emergence of a small peak, but did not affect the overall results, still less than $200 \mathrm{r} / \mathrm{min}$ when the phosphorus release volume, that disturbance intensity and phosphorus release is not showed a positive correlation. It also verifies the experimental results of Cai Jingbo ${ }^{[5]}$ et al. At low speed, the effect of mixing and exchange of sediment and water on phosphorus release from sediments is more important than that of pure dissolved oxygen. At the same time, the disturbance makes the phosphorus in the water diffusion in the water, and the results of the water exchange between the overlying water and the water gap will increase the $\mathrm{pH}$ value of the gap water, and promote more phosphorus from the solid phase into the gap water. In 200r/min, the maximum release amount is in a middle position, which is similar to the normal distribution.

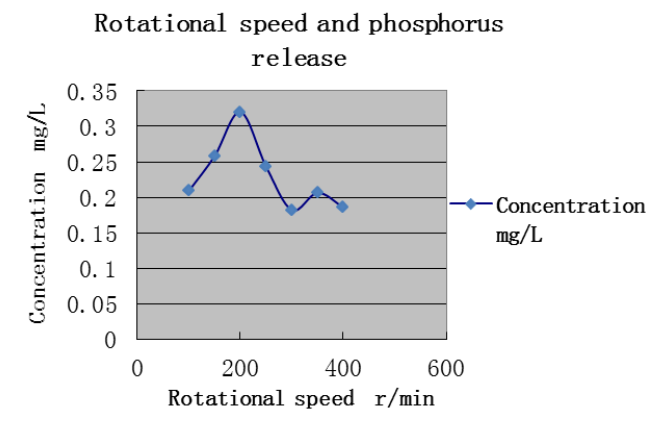

Figure2. Rotational speed and phosphorus release

\subsection{Influence of disturbance time on the release of phosphorus from sediments}

Two times respectively in HAOHE North Bridge, and HAOHE river People's Park Bridge of time series, the standard curve respectively: $\mathrm{y}=0.0234 \mathrm{x}+1.0 \mathrm{E}-4$ $\mathrm{R}^{2}=0.9999 ; \mathrm{y}=0.0202 \mathrm{x}-0.0031 \mathrm{R}^{2}=0.9998$. The curve is highly consistent. The results show that the release of phosphorus had a certain rule to follow in time, which was in the form of wave type. From Figure 3, figure 4, it is clear that the phosphorus in the sediments did not release to the aqueous solution. On the contrary, phosphorus in water solution back to the sediments and in time sequence in 72 hours to release at least, return to the sediment deposition in the most. That is to say, even if disturbed, it does not mean that the disturbance time more long, the release amount of the greater. It is released at 24 hours the most, that is, back to the sediment in the sediment at least. It can be seen that the phosphorus in the sediment did not show a "source" of the state, but rather the contrary, showing a "sink" of the state. And the first time series in December 2013 and January 2014 completed, and in March 2014 the results of the curve is quite consistent. It shows that the temperature for the experiment did not play a control and affect the conditions.

Preliminary can draw the following conclusions: in 24 hours, both the static release and dynamic release, the phosphorus release is the most. That is exactly the mud at least. And in 72 hours, whether it is static or dynamic, phosphorus release is at least and bottom mud in the most. Time series experiments results show that the phosphorus release amount and not with increase of time, the release quantity also increases, but after the release of the day release up to the amount of. Also is to say, if people for shallow lakes produced some disturbance, then at the same time the next day can phosphorus release is the largest, and relevant departments need to make a deal, reduce the nutrition level and then reduce the harm. 


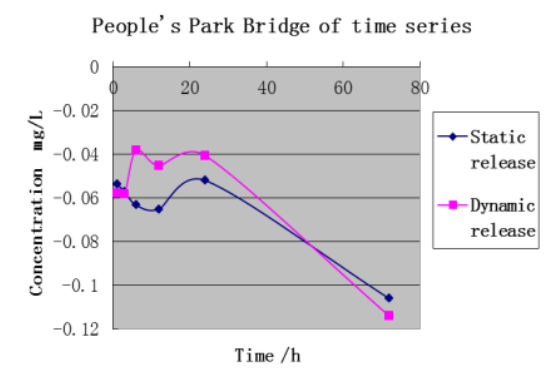

Figure3. People's Park Bridge of time series

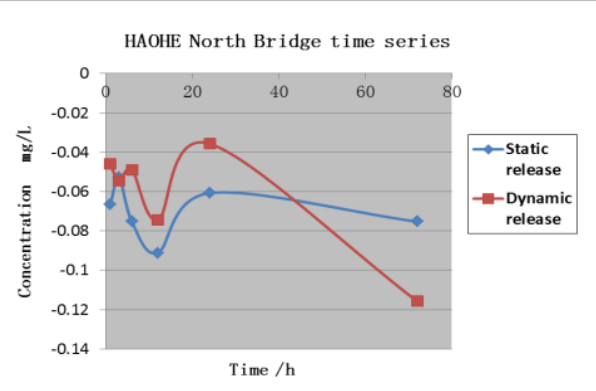

Figure4. HAOHE North Bridge of time series

\subsection{Effects of disturbance depth on the release of phosphorus from sediments}

Two times respectively in HAOHE North Bridge, HAOHE River People's Park Bridge the depth sequence, the standard curve are $\mathrm{y}=0.0202 \mathrm{x}-0.0031 \mathrm{R}^{2}=0.9998$ ). By the following two pieces of test depth Figure 5 and figure 6 that: whether it is static or dynamic phosphorus do not release the, but return to the sediment, showing a "sink" state. Especially in $15 \mathrm{~cm}$ performance is particularly evident, showing the shape of inverted triangle, breaking the conventional conclusion, is not closer to the sediment, phosphorus release quantity is more, in the $10 \mathrm{~cm}$ and $25 \mathrm{~cm}$ of the static and dynamic are in a state of high release. And the minimum value is at $15 \mathrm{~cm}$. Once emerging the disturbance, the most close to the bottom of the sediment and the closest to the water is the largest phosphorus release. And in the middle of the depth of the release of phosphorus is less, the more light is rich in nutrition. Perturbation due to simulate sediment thickness range covering the surface of the water with a certain distance on the surface of phosphorus release produced little effect. It can provide the references for the relevant pollution-control sections, also can increase the depth of the water to reduce endogenous pollution.
People's Park Bridge of depth series

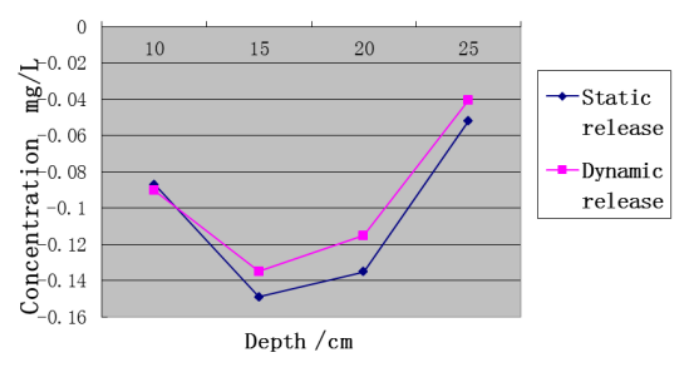

Figure5. People's Park Bridge of depth series

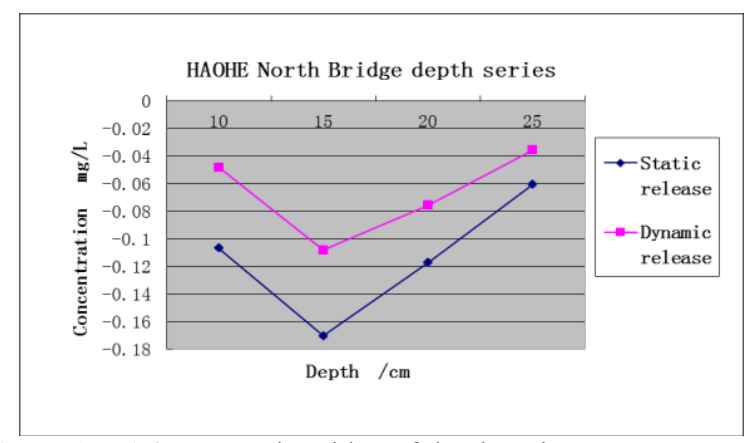

Figure6. HAOHE North Bridge of depth series

\section{Conclusions}

A. Experimental study shows that the external disturbance can significantly increase the release of endogenous phosphorus in sediment. The high speed disturbance results in the internal phosphorus release being not higher than the low velocity disturbance. Instead the distribution is approximately an approximate distribution of the intermediate disturbance velocity. In the static state of the water body, the internal phosphorus is from low concentration to high concentration of the transference process. And the disturbance is to speed up the transfer process, and also promote the release of phosphorus.

B. Overall, sometimes the role of the disturbance is not "promote" the release of endogenous sediment, that is, not "source", but a "sink" of the state. It owes to the disturbance of the water to strengthen the disturbed state. Make the phosphorus in a high speed scattered, suspended state, but makes it more conducive to the sediment for phosphorus adsorption. Or the perturbation increases the low gradient of dissolved oxygen, $\mathrm{PH}$ changes, so that the phosphorus is adsorbed by the sediment easily. From this view, the "sink" and "source" phenomenon exists at the same time, and also plays a role. C. From the time series of the experimental results, the release of phosphorus is not increased with the increase in time. But after the release of the day release most. That is to say, if people for shallow lakes produced some disturbance, then in the second day of the same time phosphorus release is the most. The relevant departments to make a certain processing, reduce the nutrition level and at last reduce the harm.

D. Deep test, once appear the disturbance situation, the most close to the bottom of the sediment and the closest 
to the water for maximum phosphorus release. And in the middle of the depth of the phosphorus release is less and the nutrition is lighter. Because there is a certain distance between the mud depth and the water surface. Distance can result in a little effect on the phosphorus release from water surface. It can provide the basis for the relevant pollution control department. It also means that some relevant departments can increase the depth of the water to reduce endogenous pollution.

E. Follow-up researchers should sum up previous experience and put up new ideas. And constantly explore new knowledge, improve methods and strategies to establish a more complete, scientific research system or model. So as to we can find a more useful method to control the release of sediment phosphorus. And then control the eutrophication of the water body.

\section{References}

1. K. Kruusement, J.M. Punning, Proc. Estonian Acad. Sci. Biol. Ecol 49, 163 (2000)

2. G.W. Zhu, B.Q. Qin, G. Gao, Chin. Sci. Bull 01, 66 (2005)

3. D.P. Li, Y. Huang, Chin. Wt. Waste 04, 17 (2013)

4. B. Yang, X.B. Yin, Jiangsu. EST 20, 61 (2007)

5. J.B. Cai, X.F. Ding, H.Y. Peng, H.Q. Chang, X.E. Yang, P.M. Pu, J. Soil. Wt. Conserv 02, 151 (2007) 